\title{
Grand canonical Monte Carlo simulations of chain molecules: adsorption isotherms of alkanes in zeolites
}

\author{
By BEREND SMIT \\ Shell Research B.V., Koninklijke/Shell-Laboratorium, Amsterdam, \\ P.O. Box 38000, 1030 BN Amsterdam, The Netherlands
}

(Received 9 November 1994; revised version accepted 26 January 1995)

Simulations of open systems are performed conveniently in the grand canonical ensemble. For chain molecules simulations of this type converge very poorly because of the very low probability of a successful insertion in the exchange step. Here, it is shown that the recently developed configurational-bias Monte Carlo technique can be used in a grand canonical Monte Carlo simulation to make the insertion of chain molecules possible. The use of this technique is illustrated by calculations of the adsorption isotherms of butane and hexane in the zeolite silicate.

\section{Introduction}

Most molecular simulations are performed either in the microcanonical ensemble (molecular dynamics) or in the canonical ensemble (Monte Carlo). In the microcanonical ensemble the thermodynamic variables that are kept constant are the temperature, volume, and total energy, whereas in the canonical ensemble the temperature, volume, and number of particles are fixed [1]. To determine an adsorption isotherm (in our case for a zeolite) we have to know the number of particles inside the zeolite as a function of the pressure and temperature of the reservoir which is in contact with the zeolite. A naive, but theoretically valid approach would be to use the molecular dynamics technique (microcanonical ensemble) and simulate the experimental situation: an adsorbent in contact with a gas (see figure 1). Such a simulation is possible only for very simple systems. In real life experiments equilibration may take minutes or even several hours, depending on the type of gas molecule. These equilibration times would be reflected in a molecular dynamics simulation, the difference being that a minute of experimental time takes of the order of $10^{9}$ seconds on a computer. In most cases we are not interested in the properties of the gas phase, yet a significant amount of CPU time will be spent on the simulation of this phase. Furthermore, in such a simulation there is an interface between the gas phase and the zeolite. In this interfacial region the properties of the system are different from the bulk properties in which we are interested. Since in a simulation the systems are small compared with experiment (hence the interfacial region is a relatively large part of such a system), we have to simulate an unnecessarily large system to minimize the influence of this interfacial region. Such a simulation would, of course, be appropriate if the interest lies in just this region.

Most of these problems can be solved by a careful choice of ensembles. For adsorption studies a natural ensemble to use is the grand canonical ensemble (or $\mu$, $V, T$ emsemble). In this ensemble the temperature, volume, and chemical potential are fixed. In the experimental setup the adsorbed gas is in equilibrium with the gas 


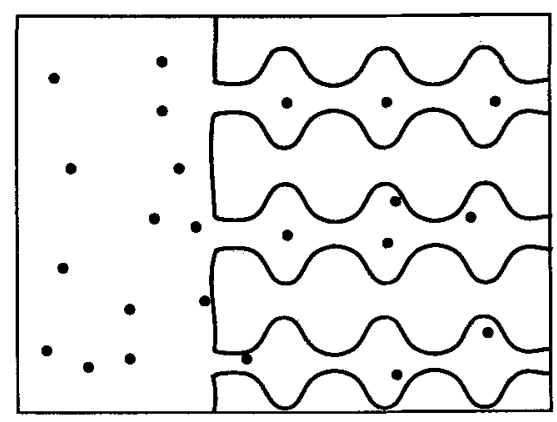

Figure 1. A zeolite in direct contact with a gas.

in the reservoir. The equilibrium conditions are that the temperature and chemical potential of the gas inside and outside the zeolite must be equal. (Note that pressure is not defined inside the zeolite, therefore pressure cannot be an equilibrium quantity. However, the pressure is related to the chemical potential via an equation of state, and it is always possible to calculate the pressure of the gas that corresponds to a given chemical potential and vice versa.) The gas that is in contact with the adsorbent can be considered as a reservoir that imposes a temperature and chemical potential on the adsorbed gas (see figure 2). We therefore have to know only the temperature and chemical potential of this reservoir to determine the equilibrium concentration inside the adsorbent. This is exactly what is mimicked in the grand canonical ensemble; the temperature and chemical potential are imposed and the number of particles is allowed to fluctuate during the simulation. This makes these simulations different from the conventional ensembles where the number of molecules is fixed.

The grand canonical Monte Carlo method works best if the acceptance of trial moves by which particles are added or removed is not too low. For atomic fluids this condition effectively limits the maximum density at which the method can be used to about twice the critical density. Special tricks are needed to extend the grand canonical Monte Carlo method to somewhat higher densities [2]. The grand canonical Monte Carlo technique is implemented easily for mixtures and inhomogeneous systems, such as fluids near interfaces. In fact, some of the most useful applications of the grand canonical Monte Carlo method are precisely in these areas of research. For more details of the grand canonical Monte Carlo method, the reader is referred to the book by Allen and Tildesley [1] and a review paper by Frenkel [3]. Although the grand canonical Monte Carlo method can be applied to simple

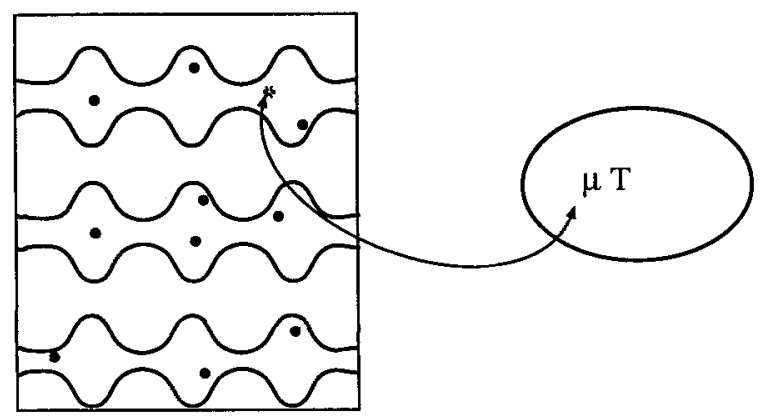

Figure 2. A zeolite in contact with a reservoir that imposes constant chemical potential and temperature by exchanging particles and energy. 
models of non-spherical molecules, special techniques are required since the method converges very poorly for all but the smallest polyatomic molecules.

In order to visualize this, consider, for example, the adsorption of alkanes in a zeolite. The bottleneck in the grand canonical Monte Carlo scheme is the particle insertion step. The probability that we accept a particle that has been placed at a random position depends on its energy. From acceptance rule (4) defined later it follows that this probability is high when the energy of the particle is low and the probability of success is low when its energy is high. Clearly, if a part of a molecule overlaps with one of the zeolite atoms, the probability of acceptance is zero. For methane, the probability that we select a position that does not give an overlap is of the order of 1 in $10^{3}$. For ethane, we have to find two positions that do not overlap, and if we assume that these positions are independent, the probability of success will be 1 in $10^{6}$. Clearly, for long-chain alkanes the probability of a successful insertion is so low that in order to obtain a reasonable acceptance the number of attempts needs to be prohibitively large.

The configurational-bias Monte Carlo technique has been developed to make possible the insertion of long-chain molecules in moderately dense liquids. The original configurational-bias Monte Carlo technique has been developed for lattice models $[4,5]$ and has been extended to continuous models $[6,7]$. Here it is shown that the configurational-bias Monte Carlo technique can also be used in the grand canonical ensemble.

\section{Grand canonical Monte Carlo}

\subsection{Preliminaries}

The grand canonical Monte Carlo method was first developed for classical fluids by Norman and Filinov [8], and later extended and improved by a number of other groups $[2,9-15]$. For our purpose it is convenient to use the derivation of Frenkel [3] to introduce the statistical mechanical basis for the grand canonical Monte Carlo technique. Let us consider a system which is a combination of two systems; a system with volume $V$ in which the $N$ molecules interact and a system with volume $V-V_{0}$ in which the $m=N-M$ molecules behave like an ideal gas (see figure 3). The partition function of this combined system is

$$
Q\left(N, m, V, V_{0}, T\right)=\frac{V^{N}\left(V_{0}-V\right)^{m}}{\Lambda^{3 M} N ! m !} \int \mathrm{d} s^{m} \int \mathrm{d} s^{N} \exp \left[-\beta U\left(s^{N}\right)\right]
$$

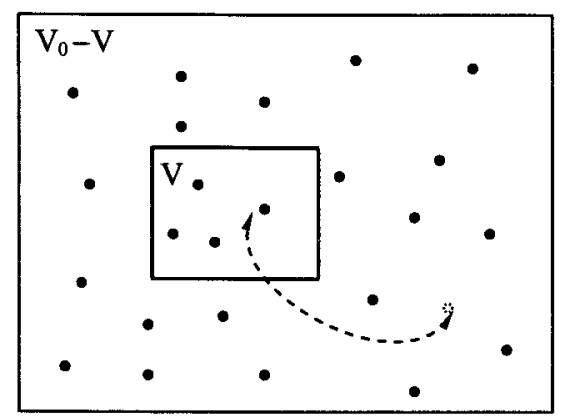

Figure 3. Ideal gas ( $m$ particles, volume $V_{0}-V$ ) that can exchange particles with an $N$-particle system (volume $V$ ). 
where $A$ is the thermal de Broglie wavelength, $\beta=1 / k_{\mathrm{B}} T$, and $s^{N}=r^{N} / L$ are the coordinates scaled with box length $L$. Now, let us see what happens when the systems exchange particles (see figure 3). To be more precise, we assume that the molecules in the two sub-volumes are actually identical particles. The only difference is that they interact when they are in volume $V$ but not when they are in volume $V_{0}-V$. If we transfer a molecule $i$ from a reduced coordinate $s_{i}$ in volume $V_{0}-V$ to the same reduced coordinate in volume $V$, then the potential energy function $U$ changes from $U\left(s^{N}\right)$ to $U\left(s^{N+1}\right)$. The expression for the total partition function of the system, including all possible distributions of the $M$ particles over the two sub-volumes, is

$$
Q\left(M, V, V_{0}, T\right)=\sum_{N=0}^{M} \frac{V^{N}\left(V_{0}-V\right)^{M-N}}{A^{3 M} N !(M-N) !} \int \mathrm{d} s^{M-N} \int \mathrm{d} s^{N} \exp \left[-\beta U\left(s^{N}\right)\right]
$$

We now write the probability density to find a system with $M-N$ particles at reduced coordinates $\boldsymbol{s}^{M-N}$ in volume $V^{\prime} \equiv V_{0}-V$ and $N$ particles at reduced coordinates $\boldsymbol{s}^{N}$ in volume $V$ as

$$
\mathscr{N}\left(s^{M} ; N\right)=\frac{V^{N} V^{M-N}}{Q\left(M, V, V^{\prime}, T\right) \Lambda^{3 M} N !(M-N) !} \exp \left[-\beta U\left(s^{N}\right)\right]
$$

Let us now consider a trial move in which a particle is transferred from $V^{\prime}$ to $V$. First we should make sure that we construct an underlying Markov process that is symmetric. Symmetry, in this case, implies that the a priori probability of moving a particle from $V^{\prime}$ to $V$ should be equal to the $a$ priori probability of the reverse move. The probability of acceptance of a trial move in which we move a particle to or from volume $V$ is determined by the ratio of the corresponding probability densities (3):

$$
\begin{gathered}
P(N \rightarrow N+1)=\frac{V(M-N)}{V^{\prime}(N+1)} \exp \left\{-\beta\left[U\left(s^{N+1}\right)-U\left(s^{N}\right)\right]\right\} \\
P(N+1 \rightarrow N)=\frac{V^{\prime}(N+1)}{V(M-N)} \exp \left\{-\beta\left[U\left(s^{N}\right)-U\left(s^{N+1}\right)\right]\right\} .
\end{gathered}
$$

Now let us consider the limit that the ideal gas system is very much larger than the interacting system: $M \rightarrow \infty, V^{\prime} \rightarrow \infty,\left(M / V^{\prime}\right) \rightarrow \rho$. Note that for an ideal gas the chemical potential $\mu$ is related to the particle density $\rho$ by (see appendix A)

$$
\mu=k_{\mathrm{B}} T \ln \Lambda^{3} \rho .
$$

Therefore, in the limit $(M / N) \rightarrow \infty$, partition function (2) becomes

$$
Q(\mu, V, T)=\sum_{N=0}^{\infty} \frac{\exp (\beta \mu N) V^{N}}{A^{3 N} N !} \int \mathrm{d} s^{N} \exp \left[-\beta U\left(s^{N}\right)\right],
$$

and the corresponding probability density is

$$
\mathscr{N}\left(s^{N} ; N\right) \propto \frac{\exp (\beta \mu N) V^{N}}{A^{3 N} N !} \exp \left[-\beta U\left({ }^{N}\right)\right] .
$$

Equations (6) and (7) are the basic equations for grand canonical Monte Carlo simulations. Note that in these equations all explicit references to the ideal-gas system have disappeared. 


\subsection{Monte Carlo simulations}

In a grand canonical simulation, we have to sample distribution (7). There are acceptable trial moves.

1. Dispacement of particles: a particle is selected at random to give a new conformation (for example, a random displacement in the case of atoms). This move is accepted with a probability of

$$
\operatorname{acc}\left(s \rightarrow s^{\prime}\right)=\min \left(1, \exp \left\{-\beta\left[U\left(s^{N}\right)-U\left(s^{N}\right)\right]\right\}\right),
$$

(2) Insertion and removal of particles: a particle is inserted at a random position or a randomly selected particle is removed. These moves are performed with equal probability. The creation of a particle is accepted with a probability of

$$
\operatorname{acc}(N \rightarrow N+1)=\min \left[1, \frac{V}{\Lambda^{3}(N+1)} \exp \{\beta[\mu-U(N+1)+U(N)]\}\right],
$$

and the removal of a particle is accepted with a probability of

$$
\operatorname{acc}(N \rightarrow N-1)=\min \left[1, \frac{\Lambda^{3} N}{V} \exp \{-\beta[\mu+U(N-1)-U(N)]\}\right] \text {. }
$$

\subsection{Chain molecules}

In the foregoing section the principles were given of the conventional grand canonical Monte Carlo technique. Furthermore, it was argued that for long-chain molecules the probability of a successful insertion is too low to make the conventional technique feasible. Recently, we have developed the configurational-bias Monte Carlo technique $[5,6]$ in which particles are not inserted at random but grown atom by atom. This growing process introduces a bias which can be removed by adjusting the acceptance rule. The configurational-bias Monte Carlo technique has been combined successfully with the Gibbs ensemble technique [16-18]. Here, we demonstrate that the configurational-bias Monte Carlo method can be used in the grand canonical ensemble to make the insertion of long-chain alkanes possible.

\subsubsection{Preliminaries}

Although the method which is described in this section is applicable to arbitrary open systems, we introduce this method in the context of alkanes adsorbed in zeolites [19]. Zeolites are crystalline structures with well defined micropores in which molecules can adsorb.

If we were to grow an alkane molecule by placing the new atom at a random position on a sphere, most configurations would be rejected because of an intramolecular energy which is too high [18]. Therefore, for systems with strong intramolecular interactions, it is important to take these interactions into account while generating the trial conformation. The potential energy of a given conformation of an n-alkane can be divided into two contributions:

(i) the internal potential energy $\left(U^{\text {int }}\right)$, which includes the bond bending and torsion, and

(ii) the external potential energy ( $U^{\text {ext }}$, which takes into account the intermolecular interactions which have not been taken into account in the internal part (the non-bonded Lennard-Jones interactions). 


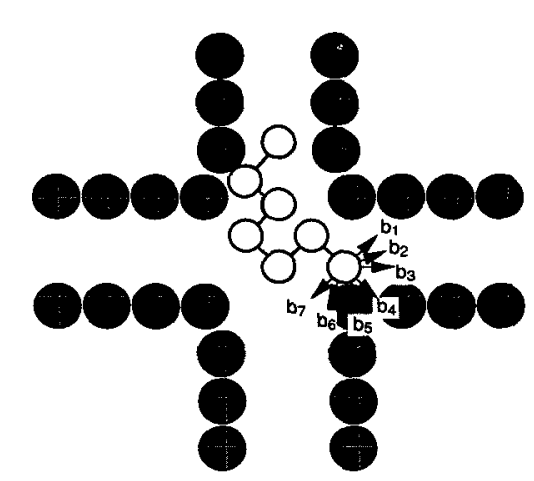

Figure 4. Schematic drawing of the growing of an alkane in a zeolite in a configurational-bias Monte Carlo move. The solid black circles represents the atoms of the zeolite and the white circles those of the alkane. The set of $k$ trial orientations $\left\{\boldsymbol{b}_{1}, \boldsymbol{b}_{2}, \ldots, \boldsymbol{b}_{k}\right)$ is indicated by arrows.

This division is to some extent arbitrary and may be optimized further. In what will follow, we refer to a chain without external interactions as an ideal chain.

\subsubsection{Particle insertion}

To insert a particle into the system, we use the following four steps.

(1) For the first atom, a random position in the zeolite is selected, and the energy of this atom is calculated (this energy is denoted by $u_{1}^{n}$ ).

(2) For the following atoms, a set of $k$ trial positions is generated. We denote these positions by $\{\boldsymbol{b}\}=\left(\boldsymbol{b}_{1}, \boldsymbol{b}_{2}, \ldots, \boldsymbol{b}_{k}\right)$ (see figure 4$)$. These positions are distributed on the surface of a sphere. The radius of this sphere is equal to the bond length and the sphere is centred around the previously inserted atom of the alkane. This set of trial orientations is generated using the internal part of the potential, which results in the following distribution for the $l$ th atom:

$$
p_{l}^{\mathrm{int}}\left(\boldsymbol{b}_{i}\right) \mathrm{d} \boldsymbol{b}_{i}=\frac{\exp \left[-\beta u_{l}^{n, \mathrm{int}}\left(\boldsymbol{b}_{i}\right)\right] \mathrm{d} \boldsymbol{b}_{i}}{C}
$$

where $\beta=1 / k_{\mathrm{B}} T$ and $C$ is a normalization constant which is not important for the simulations (see the appendix in [20]). The probability $p_{l}^{\text {int }}\left(\boldsymbol{b}_{i}\right)$ depends on which type of atom is being inserted. For the second atom, the internal potential energy is zero and as a result the trial positions will be randomly distributed on a sphere. For the third atom, the internal potential energy includes the bond bending, and for the fourth and higher atoms the internal part includes both the bond bending and torsion [20].

For each of these trial positions the external energy is calculated with the atoms of the other molecules (of the zeolite and of other alkanes) and with those atoms of the molecule that are already grown (note that this is done only if $l>4$ ). This energy is denoted by $u_{l}^{n, \operatorname{ext}}\left(b_{j}\right)$, and one of these positions is selected with a probability

$$
p_{l}^{\operatorname{ext}}\left(\boldsymbol{b}_{j}\right)=\frac{\exp \left[-\beta u_{l}^{n, \operatorname{ext}}\left(\boldsymbol{b}_{j}\right)\right]}{w^{n, \operatorname{ext}}(l)},
$$


in which

$$
w^{n, \operatorname{ext}}(l)=\sum_{j=1}^{k} \exp \left[-\beta u_{l}^{n, \operatorname{ext}}\left(b_{j}\right)\right] .
$$

Since we favour conformations with a low energy, we have introduced a bias. This bias should be removed in the acceptance rules.

(3) After repeating step 2 until the entire alkane of length $M$ has been grown, we calculate

$$
\mathscr{W}^{n} \equiv \frac{W^{n}}{k^{M-1}}=\exp \left[-\beta u^{n, \mathrm{ext}}(1)\right] \prod_{l=2}^{\mathrm{M}} \frac{w^{n, \mathrm{ext}}(l)}{k} .
$$

(4) The new alkane molecule is accepted with a probability

$$
\operatorname{acc}(N \rightarrow N+1)=\min \left(1, \frac{\exp \left(\beta \mu^{\mathbf{B}}\right) V}{\Lambda^{3}(N+1)} \mathscr{W}^{n}\right),
$$

where $\mu^{\mathrm{B}}$ is the chemical potential of a reservoir consisting of ideal chain alkanes. In appendix A it is shown how this ideal chemical potential is related to the chemical potential of a system containing interacting alkanes.

\subsubsection{Particle deletion}

To remove a particle from the system, we use the following four-stage algorithm.

(1) A particle is selected at random, the energy of the first atom is calculated and is denoted by $u^{\text {o, ext }}(1)$.

(2) For the following atoms, the external energy $u^{\mathbf{o} \text { ext }}(l)$ is calculated and a set of $k-1$ trial orientations is generated with a probability given by equation (11). Note that also in this case the probability depends on whether the second, third, fourth or higher atom is considered. Using this set of orientations and the actual position, we calculate for atom $l$

$$
w^{\text {o,ext }}(l)=\exp \left[-\beta u^{\text {o,ext }}(l)\right]+\sum_{j=2}^{k} \exp \left[-\beta u^{\text {, ext }}\left(b_{j}\right)\right] .
$$

(3) After repeating step 2 until all $M$ atoms of the alkane have been considered, we calculate

$$
\mathscr{W}^{\mathrm{o}} \equiv \frac{W^{\mathrm{o}}}{k^{M-1}}=\exp \left[-\beta u^{\mathrm{o}, \mathrm{ext}}(1)\right] \sum_{l=2}^{M} \frac{w^{\mathrm{o}, \mathrm{ext}}(l)}{k},
$$

for the entire molecule.

(4) The selected molecule is removed with a probability of

$$
\operatorname{acc}(N \rightarrow N-1)=\min \left(1, \frac{\Lambda^{3} N}{V \exp \left(\beta \mu^{\mathrm{B}}\right)} \frac{1}{\mathscr{W}^{\mathrm{o}}}\right)
$$

Note that the factor $\mathscr{W}^{\circ}$ depends on the way the old configuration is 'retraced'. If we start with atom 1 , the numerical value of $\mathscr{W}^{\circ}$ is different from the value that would be obtained if we start with atom $M$. As a consequence, the probability of such a move depends on the way the factor $\mathscr{W}^{\mathrm{o}}$ has been calculated. Such a dependence on the way a move is performed is not uncommon in a Monte Carlo simulation [20]. In appendix B it is shown that the above sampling scheme is correct, 
i.e., a Boltzmann distribution of configurations is generated if we define the chemical potential of the reservoir to be

$$
\beta \mu^{\mathrm{B}}=\beta \mu+\ln C .
$$

In appendix $\mathrm{A}$ we demonstrate that this shift in chemical potential corresponds to a change in the reference state. In an ordinary Metropolis simulation the reference state is an ideal gas, whereas in the configurational-bias scheme this reference state is an ideal chain.

\section{Applications}

To illustrate the use of the simulation technique described in this work, the adsorption isotherms of butane and hexane in the zeolite silicate have been calculated.

\subsection{Models}

The interactions of the butane and hexane molecules are described with a united atom model using the OPLS model of Jorgensen et al. [21] for butane and the model of Siepmann et al. [22] for hexane. Following the work of Kiselev and co-workers [23] the zeolite is modelled as a rigid crystal. The zeolite-alkane interactions are described using the model of June et al. [26], which gives good agreement with experimental heats of adsorption of the $\mathrm{n}$-alkanes in silicate $[24,19]$,

\subsection{Computational details}

The simulations were performed in cycles and during each cycle one of the following Monte Carlo moves was selected with a prescribed probability.

(1) Displacement of a particle. A molecule is selected at random and the entire molecule is given a random displacement in the $x, y$, or $z$ direction. The maximum displacement was set such that for each of the directions $50 \%$ of the attempted moves were accepted.

(2) Rotation of a particle. A molecule is selected at random, the entire molecule is randomly rotated, the centre of the rotation is at the centre of mass of the molecule and the axis of rotation parallel to the $x, y$, or $z$ axis. The maximum angle of rotation was set such that $50 \%$ of the attempt were accepted for any of the three axes.

(3) Partly regrowing. One of the atoms of a randomly selected molecule is shown arbitrarily and, using the configurational-bias Monte Carlo scheme, part of the molecule is regrown. The number of trial orientations for butane was five and for hexane seven.

(4) Particle exchange. It is decided at random to transfer a molecule from the system into the reservoir or to add a molecule to the system. For this move the Monte Carlo scheme outlined in this work is used.

The relative probabilities of these moves were taken as: $p_{1}: p_{2}: p_{3}: p_{4}=3: 1: 3: 3$. 


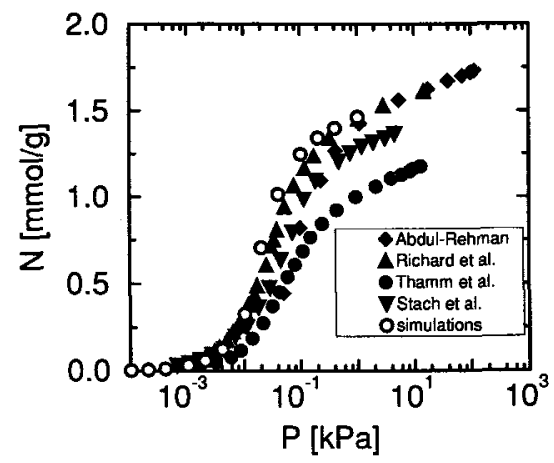

Figure 5. Adsorption isotherms: number $N$ of mmol of adsorbed butane molecules per gram of silicalite as a function of the pressure $P$ of the reservoir; the closed symbols are the experimental data of Thamm et al. [25], Stach et al. [26], Richard and Rees [27], Abdul-Rehman et al. [28], and Shen and Rees [29], and the open symbols are the results of the simulations. All the results are for $T=298 \mathrm{~K}$.

\subsection{Results}

In figure 5 the calculated adsorption isotherm of butane in silicate is compared with the experimental data of Thamm et al. [25], Stach et al. [26], Richard and Rees [27], Abdul-Rehman [28], and Shen and Rees [29]. At high pressures the data of Thamm et al. [25] deviate significantly from the majority of the reported data, possibly due to pore blocking. Considering the scatter in the experimental data the agreement between experiment and simulation is very good.

For hexane in silicate the calculated adsorption isotherm is compared in figure 6 with the experimental data as measured by Stach et al. [26], and Richard and Rees [27]. For hexane, the model of June et al. [24] overestimates the amount of adsorbed material significantly for a given pressure.

\section{Conclusion}

The bottleneck in a grand canonical Monte Carlo simulation of chain molecules is the exchange step. If the system of interest contains atoms or small molecules, the

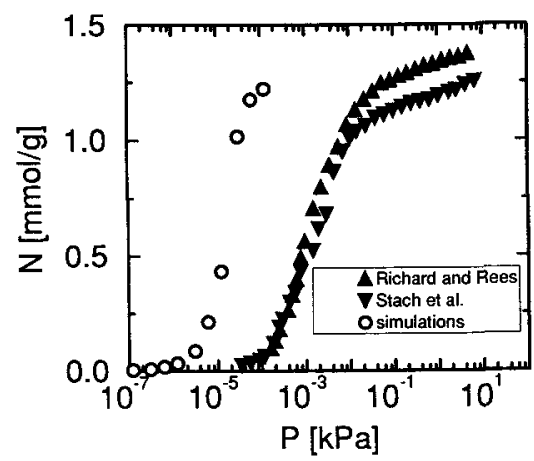

Figure 6. Adsorption isotherms of hexane: number $N$ of mmol of adsorbed hexane molecules per gram silicalite as a function of the pressure $P$ of the reservoir; the closed symbols are experimental data of Stach et al. [26], and Richard and Rees [27], and the open symbols are the results of the simulations. All results are for $T=298 \mathrm{~K}$. 
conventional grand canonical Monte Carlo method is appropriate provided that the density is not too high. For chain molecules, however, almost all attempts will result in an overlap with one of the atoms of the molecules in the system and therefore such a move has an extremely low probability of acceptance. Consequently, the conventional grand canonical Monte Carlo technique cannot be used for this type of molecule. To enable grand-canonical simulations of chain molecules, a novel Monte Carlo scheme is proposed which combines the conventional grand canonical Monte Carlo method with the configurational-bias Monte Carlo technique. In this scheme, the molecules are not inserted at random but grown atom by atom. The bias which is introduced by this growing procedure is removed subsequently by appropriate acceptance rules, which are derived in this article.

The use of this simulation technique is illustrated by the simulation of the adsorption isotherms of butane and hexane in silicalite. For butane the model of June et al. [24] yields an adsorption isotherm that is in very good agreement with experimental data. For hexane, however, the model of June et al. shows significant deviation from the experimental data.

The author would like to thank Daan Frenkel and Ilja Siepmann for their contributions to this work.

\section{Appendix A}

\section{Reference states}

If we use the configurational-bias Monte Carlo scheme to calculate the chemical potential or perform a simulation in the grand canonical ensemble, the reference state is not an ideal gas but a system containing ideal chains. In the case of alkanes, for chains longer than butane, an ideal gas chain is therefore different from an ideal chain. In this appendix, the consequences for the simulations of these differences are discussed.

\section{A.1. Definitions}

(i) Preliminaries. The partition function of a system with $N$ atoms in the $N, V, T$ ensemble is given by

$$
Z(N, V, T)=\frac{V^{N}}{\Lambda^{3 N} N !} \int \mathrm{d} s^{N} \exp \left[-\beta U\left(s^{N}\right)\right],
$$

where $s^{N}$ are the scaled coordinates of the $N$ particles. The free energy is related to the partition function via

$$
F=-\frac{1}{\beta} \ln Z(N, V, T)
$$

Using these equations we can derive the Widom expression [30] for the chemical potential

$$
\mu=-\frac{1}{\beta} \ln \left\langle\exp \left(-\beta U^{+}\right)\right\rangle+\frac{1}{\beta} \ln \left(\Lambda^{3} \rho\right),
$$

where $U^{+}$is the energy of a ghost particle (the energy of a particle that is added to the system but that does not interact with the system). For a system consisting of $N$ 
molecules with each molecule having $M$ atoms, the partition function is

$$
Z(N, M, V, T)=\frac{V^{N}}{\Lambda^{3 N} N !} \prod_{i=1}^{M} \int \mathrm{d} s_{i}^{N} \exp \left[-\beta U\left(s_{i}^{N}\right)\right] .
$$

(ii) Ideal gas. In the limit of zero density most systems behave like an ideal gas. In this limit only the intramolecular interactions contribute to the total energy, i.e.,

$$
U \approx \sum_{i=1}^{N} U^{\text {intra }}(i)
$$

For a system consisting of ideal gas atoms, partition function (A 1) reduces to

$$
Z_{\mathrm{IG}}(N, V, T)=\frac{V^{N}}{\Lambda^{3 N} N !}
$$

Using equation (A 2), we can write the chemical potential of an ideal gas of atoms as

$$
\mu_{\mathrm{IG}}=\mu_{\mathrm{IG}}^{0}+\frac{1}{\beta} \ln \rho,
$$

where the chemical potential of the reference state is defined by

$$
\mu_{\mathrm{IG}}^{0} \equiv \frac{1}{\beta} \ln A^{3} \text {. }
$$

In case of an ideal gas of molecules, the partition function (A 3) reduces to

$$
Z_{\mathrm{IG}}(N, M, V, T)=\frac{V^{N}}{\Lambda^{3 N} N !}\left\{\prod_{i=1}^{M} \int \mathrm{d} s_{i} \exp \left[-\beta U^{\text {intra }}\left(s_{i}\right)\right]\right\}^{N} .
$$

Substitution into equation (A 2) gives for the chemical potential

$$
\mu_{\mathrm{IG}}=\mu_{\mathrm{IG}}^{0}+\frac{1}{\beta} \ln \rho,
$$

where in this case the reference is defined as

$$
\beta \mu_{\mathrm{IG}}^{0} \equiv \ln \Lambda^{3}+\beta \mu_{\mathrm{intra}}^{0}=\ln \Lambda^{3}-\ln \left[\prod_{i=1}^{M} \int \mathrm{d} s_{i} \exp \left[-\beta U^{\mathrm{intra}}\left(s_{i}\right)\right]\right] .
$$

Note that the constants $\mu_{1 \mathrm{G}}^{0}$ defined by equations (A 6) and (A 9) are not important for the thermodynamics and usually can be ignored.

(iii) Ideal chain. We define an ideal chain as a chain with only internal interactions. The partition function of an ideal chain is defined by

$$
Z_{\mathrm{IC}}(N, V, T)=\frac{V^{N}}{\Lambda^{3 N} N !}\left\{\prod_{i=1}^{M} \int \mathrm{d} s_{i} \exp \left[-\beta U^{\text {int }}\left(s_{i}\right)\right]\right\}^{N},
$$

where $U^{\text {int }}$ are the internal interactions. From equations (7) and (A 10) we can derive the chemical potential of an ideal chain as

$$
\beta \mu_{\mathrm{IC}}=-\ln \left(\frac{V}{\Lambda^{3}(N+1)} \mathscr{C}\right)=\beta \mu_{\mathrm{IG}}-\ln \mathscr{C}
$$


in which the constant $\mathscr{C}$ is defined as

$$
\mathscr{C} \equiv\left\{\prod_{i=1}^{M} \int \mathrm{d} s_{i} \exp \left[-\beta u_{i}^{\mathrm{int}}\left(s_{i}\right)\right]\right\},
$$

where the sum runs over the $M$ atoms of the molecule.

The normalized Rosenbluth factor of an ideal chain, to which only the external interactions contribute, is by definition

$$
\mathscr{W}_{\mathrm{IC}}^{\mathrm{ext}} \equiv \frac{W_{\mathrm{IC}}^{\mathrm{ext}}}{k^{M-1}} \equiv 1
$$

where $k$ is the number of trial orientations.

(iv) Interacting chain. The interacting chain has both internal and external interactions

$$
U=U^{\mathrm{ext}}+U^{\mathrm{int}},
$$

and is therefore the chain in which we are interested.

\section{A.2. Rosenbluth sampling}

For an ideal gas, the normalized Rosenbluth factor can be calculated very accurately from a separate simulation. The algorithm that is used to calculate this factor is similar to the particle insertion step [31]. At each Monte Carlo step the average value of the normalized Rosenbluth factor is determined, but the molecule is never inserted. In an ideal gas, the only interactions of the molecule are with its own segments; these interactions contribute to its Rosenbluth factor.

Let us first consider the case where we have only one segment to add $(M=2)$. The probability of generating the set of $k$ trial orientations $\{\boldsymbol{b}\}_{k}$ is

$$
p^{\text {int }}\left(\boldsymbol{b}_{1}\right) \cdots p^{\text {int }}\left(\boldsymbol{b}_{k}\right) \mathrm{d} \boldsymbol{b}_{1} \cdots \mathrm{d} \boldsymbol{b}_{k}
$$

The probability of generating orientation $b$, viz. equation (1), is

$$
p^{\text {int }}(\boldsymbol{b}) \mathrm{d} \boldsymbol{b}=\frac{\exp \left[-\beta u^{\mathrm{intr}}(\boldsymbol{b})\right] \mathrm{d} \boldsymbol{b}}{C} .
$$

Since we use the algorithm of the particle insertion step, the probability of selecting a particular orientation, say $\boldsymbol{b}_{i}$, is

$$
\frac{\exp \left[-\beta u^{\text {ext }}\left(\boldsymbol{b}_{i}\right)\right]}{W^{\text {ext }}}=\frac{\exp \left[-\beta u^{\text {ext }}\left(\boldsymbol{b}_{i}\right)\right]}{\sum_{j=i}^{k} \exp \left[-\beta u^{\text {ext }}\left(b_{j}\right)\right]}
$$

To compute the avererage value of $W^{\text {ext }}$ we have to consider all orientations of the trial segments. Furthermore, each of the $k$ trial orientations has a contribution to $W^{\text {ext }}$, which is the product of the probability being selected and the value of $W^{\text {ext }}$. This gives

$$
\begin{aligned}
\left\langle\mathscr{W}_{\mathrm{IG}}^{\mathrm{ext}}\right\rangle & =\int \mathrm{d} \boldsymbol{b}_{1} \cdots \mathrm{d} \boldsymbol{b}_{k} p^{\mathrm{int}}\left(\boldsymbol{b}_{1}\right) \cdots p^{\mathrm{int}}\left(\boldsymbol{b}_{k}\right) \sum_{j=1}^{k}\left[\frac{\exp \left[-\beta u^{\text {ext }}\left(\boldsymbol{b}_{j}\right)\right]}{W^{\text {ext }}} \frac{W^{\text {ext }}}{k}\right] \\
& =\int\left[\prod_{j=1}^{k} \mathrm{~d} \boldsymbol{b}_{j} p^{\mathrm{int}}\left(\boldsymbol{b}_{i}\right)\right] \sum_{j=1}^{k} \frac{\exp \left[-\beta u^{\text {ext }}\left(\boldsymbol{b}_{j}\right)\right]}{k}
\end{aligned}
$$


Since the labelling of the trial orientations is completely arbitrary, all $k$ terms in equation (A 14) have the same contribution, and this equation can be simplified as

$$
\left\langle\mathscr{W}_{\mathrm{IG}}^{\text {ext }}\right\rangle=\int \mathrm{d} \boldsymbol{b} p^{\text {int }}(\boldsymbol{b}) \exp \left[-\beta u^{\text {ext }}(\boldsymbol{b})\right] .
$$

In case more than one segment has to be added, equation (A 15) becomes a product over all these segments [6]:

$$
\begin{aligned}
\left\langle\mathscr{W}_{\mathrm{IG}}^{\mathrm{ext}}\right\rangle & =\prod_{i=1}^{M} \int \mathrm{d} \boldsymbol{b} p_{i}^{\mathrm{int}}(\boldsymbol{b}) \exp \left[-\beta u_{i}^{\mathrm{ext}}(\boldsymbol{b})\right] \\
= & \frac{\prod_{i=1}^{M} \int \mathrm{d} \boldsymbol{b} \exp \left[-\beta u_{i}^{\mathrm{int}}(\boldsymbol{b})\right] \exp \left[\beta u_{i}^{\mathrm{ext}}(\boldsymbol{b})\right]}{\prod_{i=1}^{M} \int \mathrm{d} \boldsymbol{b} \exp \left[-u_{i}^{\mathrm{int}}(\boldsymbol{b})\right]} .
\end{aligned}
$$

Comparison with equation (A 7) shows that the numerator of equation (A 16) is equal to the configurational part partition function of one molecule in the ideal gas state, and comparison with equation (A 10) shows that the denominator is equal to the partition function of an ideal chain. The ensemble average of the Rosenbluth factor can therefore be written as

$$
\left\langle\mathscr{W}_{\mathrm{IG}}^{\mathrm{ext}}\right\rangle=\frac{Z_{\mathrm{IG}}(1, V, T)}{Z_{\mathrm{IC}}(1, V, T)},
$$

or in the terms of the chemical potentials of the ideal gas and ideal chain molecules

$$
\beta \mu_{\mathrm{IG}}-\beta \mu_{\mathrm{IC}}=-\ln \left\langle\mathscr{W}_{\mathrm{IG}}^{\mathrm{ext}}\right\rangle .
$$

Similarly, we can derive a relation between the chemical potential and the normalized Rosenbluth factor of an interacting chain:

$$
\beta \mu-\beta \mu_{\mathrm{IC}}=-\ln \left\langle\mathscr{W}^{\mathrm{ext}}\right\rangle
$$

The excess chemical potential of an interacting chain is defined as

$$
\beta \mu^{\mathrm{ex}} \equiv \beta \mu-\beta \mu_{\mathrm{IG}} \text {. }
$$

Note that in this definition we assume the reference state to be $\mu_{\mathrm{IG}}^{0}$, which is defined by equation (A 6) for a system consisting of atoms, and by equation (A 9) for a system of molecules. We can also define the excess chemical potential of an ideal chain

$$
\beta \mu_{\mathrm{IC}}^{\mathrm{ex}} \equiv \beta \mu_{\mathrm{IC}}-\beta \mu_{\mathrm{IG}}=\ln \left\langle\mathscr{W}_{\mathrm{IG}}^{\mathrm{ext}}\right\rangle .
$$

This gives for the excess chemical potential (A 20)

$$
\beta \mu_{i}^{\text {ex }}=\left(\beta \mu-\beta \mu_{\mathrm{IC}}\right)+\left(\beta \mu_{\mathrm{IC}}-\beta \mu_{\mathrm{IG}}\right)=-\ln \left\langle\mathscr{W}^{\text {ext }}\right\rangle+\beta \mu_{\mathrm{IC}}^{\mathrm{ex}}
$$

In a configurational-bias Monte Carlo simulation we can calculate the normalized Rosenbluth factor $\left\langle\mathscr{W}^{\text {ext }}\right\rangle$. Equation (A 22) shows we can use this factor to calculate an excess chemical potential which is shifted with respect to the 'real' chemical potential. To calculate this shift we need to perform a separate simulation for one molecule in the ideal gas state. Note that normally such an ideal gas simulation has to be performed only once at a given temperature; furthermore, such a simulation requires only a fraction of the CPU time of a simulation with an interacting chain. 


\section{A.3. Thermodynamic quantities}

In the previous section we have shown that the use of the ideal chain as a reference state results in a shift of the chemical potential. Below we show how this shift should be taken into account in the calculation of the Henry coefficient and in a grand canonical Monte Carlo simulation.

A.3.1. Henry coefficient. The Henry coefficient is calculated from the adsorption isotherm using

$$
K_{\mathrm{H}} \equiv \lim _{P \rightarrow 0} \frac{\langle N\rangle}{P V} .
$$

The Henry coefficient can also be related to chemical potential. For an open system in contact with a reservoir with chemical potential $\mu^{\mathbf{B}}$, the average number $\langle N\rangle$ is given by

$$
\frac{\langle N\rangle}{V}=\frac{\exp \left(\beta \mu^{\mathrm{B}}\right)}{\Lambda^{3}}\left\langle\exp \left(-\beta U^{+}\right)\right\rangle
$$

where $U^{+}$represents the energy of a ghost particle. In the limit $P \rightarrow 0$, the reservoir can be considered as an ideal gas. Substitution of the gas law in equation (A 5) gives

$$
\mu^{\mathbf{B}}=\mu_{\mathrm{IG}}^{0}+\frac{1}{\beta} \ln (\beta P) .
$$

Using the definition of the excess chemical potential (A 20) and equation (A 22) we can write

$$
\left\langle\exp \left(-\beta U^{+}\right)\right\rangle=\exp \left(-\beta \mu^{\mathrm{ex}}\right)=\left\langle\mathscr{W}^{\mathrm{ext}}\right\rangle \exp \left(-\beta \mu_{\mathrm{IC}}^{\mathrm{ex}}\right)
$$

Substitution of equations (A 24) and (A 25) into (A 23) gives

$$
K_{\mathrm{H}}=\frac{\langle N\rangle}{V P}=\beta\left\langle\mathscr{W}^{\mathrm{ext}}\right\rangle \exp \left(-\beta \mu_{\mathrm{IC}}^{\mathrm{ex}}\right\rangle
$$

\section{A.3.2. Grand canonical calculations}

(a) Simple atomic system. In the grand canonical ensemble the partition function for a system consisting of $N$ atoms is given by equation (6) and the distribution that has to be sampled is given by equation (7). To sample this distribution, we have used the following acceptance rules in the conventional grand canonical Monte Carlo scheme:

$$
\operatorname{acc}(N \rightarrow N+1)=\min \left[1, \frac{V}{\Lambda^{3}(N+1)} \exp \left\{\beta\left[\mu^{\mathbf{B}}-U(N+1)+U(N)\right]\right\}\right],
$$

for the addition of a particle (see equation (9)) and

$$
\operatorname{acc}(N \rightarrow N-1)=\min \left[1, \frac{A^{3} N}{V} \exp \left\{-\beta\left[\mu^{\mathbf{B}}+U(N-1)-U(N)\right]\right\}\right]
$$

for the removal of a particle (see equation (10)).

These equations are based on the idea that particles are exchanged with a reservoir consisting of the same molecules with exactly the same density, the only difference being that in the reservoir the particles behave like an ideal gas; hence we are 
equating the chemical potential $\mu^{\mathbf{B}}$ in this reservoir and the chemical potential of the system we are simulating. The chemical potential of the reservoir is related to the ideal gas pressure by:

$$
\beta \mu^{\mathrm{B}} \equiv \beta \mu_{\mathrm{IG}}=\beta \mu_{\mathrm{IG}}^{0}+\ln (\rho)=\beta \mu_{\mathrm{IG}}^{0}+\ln \left(\beta P_{\mathrm{IG}}\right) .
$$

Substitution of this expression in the acceptance rules gives (for the addition of a particle):

$$
\operatorname{acc}(N \rightarrow N+1)=\min \left[1, \frac{V \beta P_{\mathrm{IG}}}{(N+1)} \exp \{-\beta[U(N+1)-U(N)]\}\right] .
$$

If the density in the 'experimental reservoir' is sufficiently low such that it behaves like an ideal gas then the pressure $P$ of the reservoir will be equal to the pressure $P_{\mathrm{IG}}$ of the reservoir (the chemical potentials are by definition always equal!).

If the pressure in the reservoir is too high for the ideal gas law to hold, then we have to use the equation of state to relate the chemical potential of the reservoir to the pressure:

$$
\begin{gathered}
\beta \mu_{\text {bath }}=\beta \mu_{\text {reservoir }} \\
\beta \mu_{\mathrm{IG}}^{0}+\ln \left(\beta P_{\mathrm{IG}}\right)=\beta \mu_{\mathrm{IG}}^{0}+\ln (\beta P / \phi),
\end{gathered}
$$

where $\phi$ is the fugacity coefficient of the fluid in the reservoir, which can be calculated from the equation of state of the fluid.

(b) Molecular system. For a system containing molecules we also use equation (A 28) for the acceptance rule. However, its relation to the chemical potential is slightly different. For such systems the reference chemical potential is defined by equation (A 6). Comparison with equation (A 27) shows that in addition to the term $\Lambda^{3}$ we have a contribution of the internal energy to the chemical potential $\mu_{\text {intra }}^{0}$ of the molecules in the ideal gas phase. We can absorb this term in the definition of $\mu^{\mathbf{B}}$, so in this case we have

$$
\mu^{\mathrm{B}}=\mu_{\mathrm{IG}}-\mu_{\mathrm{intra}}^{0} .
$$

With this equation for the chemical potential of the reservoir, we can also use equation (A 29) to calculate the pressure of the reservoir.

(c) Configurational-bias Monte Carlo. If we use the configurational-bias Monte Carlo scheme, the chemical potential of the reservoir is shifted with respect to the chemical potential used in the Metropolis algorithm:

$$
\beta \mu_{\text {CBMC }}^{\mathrm{B}}=\beta \mu^{\mathrm{B}}-\ln (\mathscr{C}) .
$$

Comparison with equation (A 11) shows that the constant $\mathscr{C}$ is related to the chemical potential of an ideal chain by

$$
\beta \mu_{\mathrm{IC}}-\beta \mu_{\mathrm{IG}}=-\ln (\mathscr{C})
$$

Since we suppose that the chemical potential in the ordinary Metropolis case is that of an ideal gas, viz. equation (A 27), it follows that

$$
\beta \mu_{\text {CBMC }}^{\mathrm{B}}=\beta \mu_{\mathrm{IG}}-\ln (\mathscr{C})=\beta \mu_{\mathrm{IC}} \text {. }
$$

This shows that if we use the configurational-bias Monte Carlo scheme, we couple our system to a reservoir consisting of ideal chain molecules. By combining 
equations (A 21) and (A 8), we can relate the chemical potential of this reservoir to the ideal gas pressure by

$$
\beta \mu_{\mathrm{CBMC}}^{\mathrm{B}}=\beta \mu_{\mathrm{IG}}+\beta \mu_{\mathrm{IC}}^{\mathrm{ex}}=\beta \mu_{\mathrm{IG}}^{0}+\ln \left(\beta P_{\mathrm{IG}}\right)+\beta \mu_{\mathrm{IC}}^{\mathrm{ex}},
$$

which shows that in this case equation (A 28) could be replaced by

$$
\operatorname{acc}(N \rightarrow N+1)=\min \left[1, \frac{V \beta P_{\mathrm{IG}}}{(N+1) \exp \left(-\beta \mu_{\mathrm{IC}}^{\mathbf{e x}}\right)} \exp \{-\beta[U(N+1)-U(N)]\}\right] \text {, }
$$

and the pressure of the reservoir follows again from equation (A 29).

\section{Appendix B}

\section{Proof of the algorithm}

\section{B.1. Metropolis Monte Carlo}

It is instructive to prove that in the case of ordinary grand canonical ensemble simulations, acceptance rules (8)-(10) indeed lead to a sampling of distribution (7). Consider a move in which we start with a configuration with $N$ particles and we move to a configuration with $N+1$ particles by inserting a particle in the system. We have to demonstrate that detailed balance is obeyed:

$$
K(N \rightarrow N+1)=K(N+1 \rightarrow N),
$$

where $K(N \rightarrow N+1)$ is the flow of configurations from a state with $N$ particles to a configuration with $N+1$ particles. This flow is the product of the probability of being in a state with $N$ particles (7), the probability of generating a state with $N+1$ particles, and the probability of accepting move (9):

$$
K(N \rightarrow N+1)=\mathscr{N}(N) \times p(N \rightarrow N+1) \times \operatorname{acc}(N \rightarrow N+1) .
$$

In the scheme of section 2.1 , the probability that an attempt is made to remove a particle is equal to the probability of attempting to add one:

$$
p(N \rightarrow N+1)=p(N+1 \rightarrow N) .
$$

Substitution of this equation together with equations (7) and (9) into the condition of detailed balance (B 1) gives as condition for the acceptance rules

$$
\begin{aligned}
\frac{\operatorname{acc}(N \rightarrow N+1)}{\operatorname{acc}(N+1 \rightarrow N)} & =\frac{\exp [\beta \mu(N+1)] V^{N+1} \exp \left[-\beta U\left(s^{N+1}\right)\right]}{\Lambda^{(N+1)}(N+1) !} \frac{\Lambda^{3 N} N ! \exp \left[\beta U\left(s^{N}\right)\right]}{\exp (\beta \mu N) V^{N}} \\
& =\frac{\exp (\beta \mu) \mathrm{V}}{\Lambda^{3}(N+1)} \exp \left\{-\beta\left[\mathrm{U}\left(s^{N+1}\right)-U\left(s^{N}\right)\right]\right\} .
\end{aligned}
$$

It is straightforward to show that acceptance rules (9) and (10) obey this condition. For the other move a similar condition can be derived which proves that the sampling scheme is correct.

\section{B.2 Configurational-bias Monte Carlo}

We now turn to the configurational-bias Monte Carlo scheme. We consider the combined system of the reservoir and our system of interacting alkanes in a zeolite. 


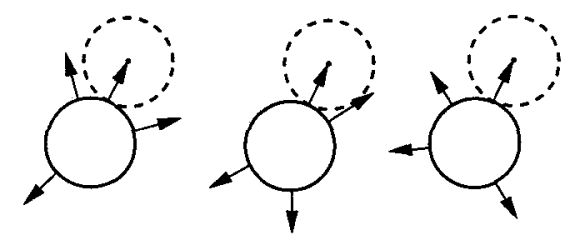

Figure B 1. An example of three sets of four-trial orientations (indicated by arrows) around the first atom. Each of these has a probability of resulting in the same configuration of the second atom (dotted sphere). Note that an infinite number of these sets exists.

A particle insertion/removal corresponds to the transfer of a molecule from the reservoir/interacting system to the interacting system/reservoir.

Let us first consider the transfer of a molecule from the reservoir into the zeolite with configuration $\Gamma$. We use the algorithm of section 2.3 .2 to generate configuration $\Gamma$ of the molecule. Note that this configuration can be generated in many ways. For example, figure B 1 shows three sets of trial orientations each of which may result in the selection of the same segment. To simplify the discussion, let us focus on the case in which we have to add only a single segment. Suppose that for this addition we use a set of $k$ trial orientations, denoted by

$$
\{\boldsymbol{b}\}_{k}=\left\{\boldsymbol{b}_{1}, \boldsymbol{b}_{2}, \ldots, \boldsymbol{b}_{k}\right\} \text {. }
$$

Conformation $\Gamma$ can be selected only if this orientation, denoted by $\boldsymbol{b}_{\Gamma}$, is in $\{\boldsymbol{b}\}_{k}$. The set of all sets $\{\boldsymbol{b}\}_{k}$ that includes this conformation is denoted by

$$
\left\{\mathscr{B}_{\Gamma}\right\}=\left\{\{\boldsymbol{b}\}_{k} \mid \boldsymbol{b}_{\Gamma} \in\{\boldsymbol{b}\}_{k}\right\} .
$$

Every element of $\left\{\mathscr{S}_{\Gamma}\right\}$ can be written as $\left(\boldsymbol{b}_{\Gamma}, \boldsymbol{b}^{*}\right)$, where $\boldsymbol{b}^{*}$ is a set of additional trial orientations. The probability of generating one of these orientations, say $b$, is given by

$$
p^{\mathrm{int}}(\boldsymbol{b}) \mathrm{d} \boldsymbol{b}=\frac{\exp \left[-\beta u^{\mathrm{int}}(\boldsymbol{b})\right] \mathrm{d} \boldsymbol{b}}{C}
$$

where the normalization constant $C$ is defined as

$$
C \equiv \int \mathrm{d} b \exp \left[-\beta u^{\mathrm{int}}(\boldsymbol{b})\right] .
$$

The probability that we generate the set $\left(b_{\Gamma}, b^{*}\right)$ is

$$
p\left(N \rightarrow N+1 \mid \boldsymbol{b}_{\Gamma}, \boldsymbol{b}^{*}\right)=k p^{\mathrm{int}}\left(\boldsymbol{b}_{\Gamma}\right) \mathrm{d} \boldsymbol{b}_{\Gamma} p^{\text {int }}\left(\boldsymbol{b}^{*}\right) \mathrm{d} \boldsymbol{b}^{*},
$$

in which the probability of generating the set of additional trial orientations is given by (viz. equation (12))

$$
p\left(\boldsymbol{b}^{*}\right) \mathrm{d} \boldsymbol{b}^{*}=(k-1) ! \prod_{j=2}^{k} p^{\mathrm{int}}\left(\boldsymbol{b}_{j}\right) \mathrm{d} \boldsymbol{b}_{j} .
$$

Note that the factors $k$ and $(k-1)$ ! in equations (B 7) and (B 8), respectively, take into account that the order of the orientations in a set $\left(b_{\Gamma}, b^{*}\right)$ is not important. The probability that we select one of these orientations, say $b_{\Gamma}$, is given by (viz. equation (12))

$$
\frac{\exp \left[-\beta u^{\mathrm{ext}}\left(\boldsymbol{b}_{\Gamma}\right)\right]}{W^{\mathrm{ext}}}
$$


Let us now demonstrate that detailed balance is obeyed by the proposed sampling scheme. Detailed balance implies that the flow of configurations in which one particle is added in configuration $\Gamma$ is equal to the reverse flow, the removal of a particle in this configuration, i.e.,

$$
K(N \rightarrow N+1 \mid \Gamma)=K(N+1 \mid \Gamma \rightarrow N),
$$

where ' $N+1 \mid \Gamma$ ' denotes that the system is in a state with $N+1$ particles in which one particle has configuration $\Gamma$, and $N$ particles have the same conformation as in the state denoted by $N$. The flow to state ' $N+1 \mid \Gamma^{\prime}$ ' is the product of the probability of being in state ' $N$ ', the probabillty of generating configuration $\Gamma$, and the probability of acceptance, considering all sets in $\left\{\mathscr{B}_{\Gamma}\right\}$ :

$$
K(N \rightarrow N+1 \mid \Gamma)=\mathscr{N}(N) \sum_{i \in\left\{\mathscr{B} \mathscr{B}_{\Gamma}\right\}} p(N \rightarrow N+1, i) \times \operatorname{acc}(N \rightarrow N+1, i) .
$$

Note that the probability of generating configuration $\Gamma$ and the acceptance depends on the particular set of trial orientations $i$.

For the reverse move, the removal of a particle, we also have to sum over the set $\left\{\mathscr{B}_{\Gamma}\right\}$ :

$$
K(N+1 \mid \Gamma \rightarrow N)=\mathscr{N}(N+1 \mid \Gamma) \sum_{j \in\left\{\mathscr{B} \mathscr{B}_{\Gamma}\right\}} p(N+1 \rightarrow N) \times \operatorname{acc}(N+1 \rightarrow N, j) .
$$

Note that in this term the set of trial orientations $j$ appears only in the acceptance probability; the probability that we attempt to remove a particle is independent of this set.

The condition of detailed balance implies that equations (B 9) and (B 10) should be equal. This condition is certainly obeyed if we impose a much stronger condition: 'super-detailed balance' [6]. Super-detailed balance states that each term on the LHS, corresponding to a particular set of trial orientations $\left(\boldsymbol{b}_{\Gamma}, \boldsymbol{b}^{*}\right)$, must be equal to its counterpart on the RHS, which is the term that has the same set of trial orientations:

$$
K\left(N \rightarrow N+1 \mid \boldsymbol{b}_{\Gamma}, \boldsymbol{b}^{*}\right)=K\left(N+1 \mid \boldsymbol{b}_{\Gamma}, \boldsymbol{b}^{*} \rightarrow N\right) .
$$

For the LHS of equation (B 11) we can write

$$
\begin{aligned}
K\left(N \rightarrow N+1 \mid \boldsymbol{b}_{\Gamma}, \boldsymbol{b}^{*}\right)= & \mathscr{N}(N) \times p\left(N \rightarrow N+1 \mid \boldsymbol{b}_{\Gamma}, \boldsymbol{b}^{*}\right) \\
& \times \operatorname{acc}\left(N \rightarrow N+1 \mid \boldsymbol{b}_{\Gamma}, \boldsymbol{b}^{*}\right),
\end{aligned}
$$

and for the RHS

$$
\begin{aligned}
K\left(N+1 \mid \boldsymbol{b}_{\Gamma}, \boldsymbol{b}^{*}\right) \rightarrow N= & \mathcal{N}(N+1 \mid \Gamma) \times p(N+1 \rightarrow N) \\
& \times \operatorname{acc}\left(N \rightarrow N+1 \mid \boldsymbol{b}_{\Gamma}, \boldsymbol{b}^{*}\right) .
\end{aligned}
$$

In the condition of super-detailed balance, the set of trial orientations for the addition of a particle is by definition equal to the one used for the removal, and therefore the normalized Rosenbluth factor calculated during the addition of a particle (equation (14)) is equal to the one calculated during the removal (equation (17)), or

$$
\mathscr{W}^{n}=\mathscr{W}^{0}=\mathscr{W}^{\mathrm{ext}}\left(\boldsymbol{b}_{\Gamma}, \boldsymbol{b}^{*}\right)
$$


Let us assume that we have selected a particular set of additional trial orientations $\left(\boldsymbol{b}_{\Gamma}, \boldsymbol{b}^{*}\right)$, and assume for this set

$$
\frac{\exp \left(\beta \mu^{\mathrm{B}}\right) V}{\Lambda^{3}(N+1)} \mathscr{W}^{\mathrm{ext}}\left(b_{\Gamma}, b^{*}\right) \leqslant 1 .
$$

Note that instead of equation (B 15) we also could have assumed

$$
\frac{\exp \left(\beta \mu^{\mathbf{B}}\right) V}{A^{3}(N+1)} \mathscr{W}^{\operatorname{ext}}\left(b_{\Gamma}, b^{*}\right)>1
$$

which would lead to the same results.

If equation (B 15) holds, then the probability of accepting the addition of a particle (equation (15)) is

$$
\operatorname{acc}\left(N \rightarrow N+1 \mid \boldsymbol{b}_{\Gamma}, \boldsymbol{b}^{*}\right)=\frac{\exp \left(\beta \mu^{\mathrm{B}}\right) V}{\Lambda^{3}(N+1)} \mathscr{W}^{\mathrm{ext}}\left(\boldsymbol{b}_{\Gamma}, \boldsymbol{b}^{*}\right),
$$

and the corresponding term for the removal of a particle (equation (18)) is

$$
\operatorname{acc}\left(N+1 \mid b_{\Gamma}, b^{*} \rightarrow N\right)=1 .
$$

Substitution of these expressions into equation (B 12) gives

$$
\begin{aligned}
K\left(N \rightarrow N+1 \mid \boldsymbol{b}_{\Gamma}, \boldsymbol{b}^{*}\right)= & \mathcal{N}(N) \times k \exp \left[\beta u^{\mathrm{ext}}\left(\boldsymbol{b}_{n}\right)\right] p^{\mathrm{int}}\left(\boldsymbol{b}_{n}\right) \mathrm{d} \boldsymbol{b}_{n} \frac{\mathrm{d} \boldsymbol{b}^{*} p^{\mathrm{int}}\left(\boldsymbol{b}^{*}\right)}{W^{\mathrm{ext}}\left(\boldsymbol{b}_{\Gamma}, \boldsymbol{b}^{*}\right)} \\
& \times \frac{\exp \left(\beta \mu^{\mathrm{B}}\right) V}{\Lambda^{3}(N+1)} \mathscr{W}^{\mathrm{ext}}\left(\boldsymbol{b}_{\Gamma}, \boldsymbol{b}^{*}\right) \\
= & \mathscr{N}(N) \frac{\exp \left(\beta \mu^{\mathrm{B}}\right) V \exp [-\beta U(\Gamma)]}{\Lambda^{\mathbf{3}}(N+1) C} \mathrm{~d} \boldsymbol{b}^{*} p^{\mathrm{int}}\left(\boldsymbol{b}^{*}\right),
\end{aligned}
$$

and for the reverse move equation (B 13), we find

$$
K\left(N+1 \mid b_{\Gamma}, b^{*} \rightarrow N\right)=\mathscr{N}(N+1 \mid \Gamma) \times \mathrm{d} b^{*} p^{\text {int }}\left(b^{*}\right) \times 1 .
$$

Note that in the algorithm for the reverse move the first trial orientation is not generated but follows from configuration $\Gamma$. From the condition of super-detailed balance (B 11), we derive for the ratio

$$
\frac{\mathscr{N}(N+1 \mid \Gamma)}{\mathscr{N}(N)}=\frac{\exp \left(\beta \mu^{\mathbf{B}}\right) V \exp [-\beta U(\Gamma)]}{A^{3}(N+1) C} .
$$

Comparison with equation (7) shows that our sampling scheme generates the desired distribution if we define the chemical potential of the reservoir to be

$$
\beta \mu^{\mathbf{B}}=\beta \mu+\ln C .
$$

In appendix $\mathrm{A}$ we demonstrated that this shift in chemical potential corresponds to a change in the reference state. In an ordinary Metropolis simulation the reference state is an ideal gas whereas in the configurational-bias scheme this reference state is an ideal chain. 


\section{References}

[1] Allen, M. P., and Tildesley, D. J., 1987, Computer Simulation of Liquids (Oxford: Clarendon Press).

[2] Mezei, M., 1980, Molec. Phys., 40, 901.

[3] Frenkel, D., 1986, Molecular Dynamics Simulations of Statistical Mechanics Systems, edited by G. Ciccotti and W. G. Hoover, p. 151 (Ansterdam: North-Holland).

[4] Harris, J., and Rice, S. A., 1988, J. chem. Phys., 89, 5898.

[5] Siepmann, J. I., and Frenkel, D., 1992, Molec. Phys., 75, 59.

[6] Frenkel, D., MoouJ, G. C. A. M., and Smit, B., 1992, J. Phys.: Condens. Matter, 4, 3053.

[7] de Pablo, J. J., Laso, M., and Suter, U. W., 1992, J. chem. Phys., 96, 2395.

[8] Norman, G. E., and Filinov, V. S., 1969, High Temp. (USSR), 7, 216.

[9] Adams, D. J. 1974, Molec. Phys., 28, 1241.

[10] Adams, D. J., 1975, Molec. Phys., 29, 307.

[11] Adams, D. J., 1976, Molec. Phys., 32, 647.

[12] Adams, D. J., 1979, Molec. Phys., 37, 211.

[13] Yao, J., Greenkorn, R. A., and Chao, K. C., 1982, Molec. Phys., $46,587$.

[14] Valleau, J. P., and Cohen, L. K., 1980, J. chem. Phys., 72, 5935.

[15] Van Megen, W., and Snook, I. K., 1980, J. chem. Phys., 73, 4656

[16] MooiJ, G. C. A. M., Frenkel, D., and SMIT, B., 1992, J. Phys.: condens. Matter, 4, L255.

[17] Laso, M., De Pablo, J. J., and Suter, U. W., 1992, J. chem. Phys., 97, 2817.

[18] Smit, B., Karaborni, S., and Siepmann, J. I., 1995, J. chem. Phys., 102, 2126

[19] SMit, B., and SiePMANN, J. I., 1994, Science, 264, 1118.

[20] Smit, B., and Siepmann, J. I., 1994, J. phys. Chem., 98, 8442.

[21] Jorgensen, W. L., Madura, J. D., and Swenson, C. J., 1984, J. Amer. chem. Soc., 106, 6638.

[22] Siepmann, J. I., Karaborni, S., and Smit, B., 1993, Nature, 365, 330.

[23] Bezus, A. G., Kiselev, A. V., Lopatkin, A. A., and Du, P. Q., 1978, J. chem. Soc. Faraday Trans. ii 74, 367.

[24] June, R. L., Bell, A. T., and Theodorou, D. N., 1992, J. phys. Chem., 96, 1051.

[25] Thamm, H., Stach, H., SChIRMER, W., and FahlKe, B., 1982, Z. phys. Chem., 263, 461.

[26] Stach, H., Lohse, U., Thamm, H., and SChIRmer, W., 1986, Zeolites, 6, 74.

[27] Richard, R. E., and ReES, L. V. C., 1987, Langmuir, 3, 335.

[28] Abdul-Rehman, H. B., Hasanain, M. A., and Loughlin, K. F., 1990, Ind enging Chem. Res., 29, 1525.

[29] ShEn, D., and Refs, L. V., 1991, Zeolites, 119, 684.

[30] Widom, B., 1963, J. chem. Phys., 39, 2802.

[31] Frenkel, D., and Smit, B., 1992, Molec. Phys., 75, 983. 